\title{
Airway neutrophils and interleukin-17
}

\author{
A. Lindén, H. Hoshino, M. Laan
}

\begin{abstract}
Airway neutrophils and interleukin-17. A. Lindén, H. Hoshino, M. Laan. (C)ERS Journals Ltd 2000.
\end{abstract}

ABSTRACT: It is well known that exacerbations of obstructive airways disease such as asthma and chronic obstructive pulmonary disease are associated with an increased number of neutrophils in the airways. However, the mechanisms behind this phenomenon are poorly understood.

There is in vivo experimental evidence that the number of airway neutrophilis is controlled by certain T-lymphocytes, but the mediators responsible for this lymphocyte-related neutrophilia have not yet been identified.

In this review, novel evidence that the T-lymphocyte-related cytokine interleukin (IL)-17 can link the activation of certain T-lymphocytes to the recruitment and activation of airway neutrophils is described. The IL-17-induced neutrophil recruitment is mediated via induced CXC chemokine release through steroid-sensitive mechanisms and is modulated by release of endogenous tachykinins. These effects of IL-17 are potentiated by other pro-inflammatory cytokines such as (IL-1 $\beta$ ) and tumour necrosis factor- $\alpha$.

Clinical studies are needed to evaluate whether or not targeting these mechanisms can provide a useful pharmacotherapeutical approach against exaggerated mobilization of neutrophils in obstructive airways disease.

Eur Respir J 2000; 15: 973-977.
Dept of Respiratory Medicine \& Allergology, Gothenburg, Sweden.

Correspondence: A. Lindén, Lung Pharmacology Group, Dept of Respiratory Medicine \& Allergology, Guldhedsgatan 10A, SE-413 46 Gothenburg, Sweden. Fax: 4631413290

Keywords: Airway, cytokine, elastase, interleukin-8, myeloperoxidase, neutrophil

Received: June 251999

Accepted after revision December 301999

This work was funded by Hermann Krefting's Foundation, the Swedish Medical Research Council(K97-04X-09-04-8-08A, U1268), the Vardal Foundation and the Swedish Heart Lung Foundation. No support, direct or indirect, was obtained from the tobacco industry.
Exacerbations of obstructive airways diseases such as asthma and chronic obstructive pulmonary disease (COPD) may be associated with an increased number of neutrophilic granulocytes in the airways. This has been indicated by studies involving bronchoalveolar lavage (BAL) fluid and bronchial biopsy samples as well as induced sputum from patients [1-6]. Recent data suggest that this increased number of airway neutrophils is not associated with detectable airway infection [6].

Neutrophils are capable of releasing mediators which cause effects that resemble exacerbations of obstructive airways disease. For example, neutrophils can release enzymes such as neutrophil elastase [7, 8], a serine protease that degrades elastin and exerts a potent secretagogue effect on airway gland cells $[8,9]$, and may thus contribute to lung tissue damage and to airway gland hypersecretion respectively $[8,10]$. Neutrophils can also produce oxygen free radicals, which cause increased transcription of the messenger ribonucleic acid (mRNA) for the potent neutrophil chemoattractant interleukin (IL)-8 in bronchial epithelial cells [11-13]. Furthermore, neutrophils can release IL-8 in response to eosinophil granule major basic protein [14], which is of interest because the number of eosinophils, just as the IL-8 level, is often increased in asthmatic airways $[5,15]$. In addition to this, neutrophils can release the pro-inflammatory cytokine tumour necrosis factor- $\alpha$ (TNF- $\alpha)$ [16], which is increased in obstructive airways disease [15], and this cytokine can cause bronchial smooth muscle hyperresponsiveness [17], possibly through the release of the CXC chemokine IL-8 from bronchial epithelial cells [17-19]. The recruitment and activation of neutrophils in the airways may therefore contribute to excacerbations of obstructive airways disease. This also means that factors controlling the mediators affecting neutrophil recruitment, such as CXC chemokines or tachykinins $[12,13,20]$, may play a role in triggering exacerbations of obstructive airways disease.

\section{The role of T-lymphocytes}

It is likely that T-lymphocytes of the CD4+ subset play a central role in the recruitment of granulocytes into the airway lumen in obstructive airways disease [2, 21-23]. For asthma, this is supported by the observation that specific antibodies directed against CD4+ cells inhibit allergen-induced influx of eosinophils and neutrophils into rodent airways in vivo and so do anti-IL-2 receptor antibodies [23, 24], whereas it remains to be proven that this is the case in COPD. It is also noteworthy that neutrophils contain defensins that are capable of recruiting and modulating T-lymphocytes [8], thereby providing a possible feedback mechanism between CD4+ cells and neutrophils. Whereas there is now massive evidence that IL-5 released from $\mathrm{CD} 4+$ cells plays a role in mediating allergen-induced eosinophil recruitment into asthmatic airways [25], the mediator linking the activation of CD4+ cells to neutrophil recruitment has not yet been identified. IL-17 may be a candidate. 


\section{The interleukin-17 molecule}

IL-17 is a homodimeric protein comprising 23 amino acids and has a molecular weight ranging 15-22 kDa [24]. Rat (r), mouse (m) and human (h) IL-17 display a high degree of structural homology [24]. This homology includes the functionally important glycosylation site [24].

Certain human and murine T-lymphocytes of the CD4+ subset can produce and release IL-17 when activated in vitro [26-28]. Thus, in patients with rheumatoid arthritis, IL-17 can be released from CD4+ cells isolated from synovial tissue and fluid in vitro and it appears that IL-17 is produced mainly by the T-helper (Th) 0 and Th1 subsets of these cells [29]. In isolated human peripheral blood mononuclear cells in vitro, $\mathrm{CD} 4+$ as well as CD8+ memory T-cells (CD45RO) express IL-17 mRNA under certain conditions [30], but the quantity of IL-17 released from CD4+ cells probably exceeds that from CD8+ cells [27]. IL-17 is probably not expressed constitutively under physiological conditions [26].

\section{Interleukin-17 receptors}

Compared with other known receptor families, the receptor for IL-17 displays a unique structure [26, 28, 31]. The mRNA for the hIL-17 receptor is expressed in several human cell types, such as a lung epithelial cell line, foreskin fibroblasts, a B-cell line, a myelomonocytic cell line and a embryonal kidney cell line [31]. Several of these cell types express the hIL-17 receptor constitutively.

The mRNA for the mIL-17 receptor is distributed in several tissues including the lungs in mice [32]. When cultured in vitro, fibroblast, intestinal epithelial and Tlymphocyte cell lines from mice also express mRNA for the mIL-17 receptor [32].

\section{Interleukin-17 causes the release of neutrophil- modulating cytokines}

Stimulation by hIL-17 causes human synovial fibroblasts to release IL-8 and granulocyte colony-stimulating factor in human synovial fibroblasts cultured in vitro [33]. Costimulation by hIL-17 together with hTNF- $\alpha$ results in release of granulocyte-macrophage colony-stimulating factor in these synovial fibroblasts. In response to IL-17, human foreskin fibroblasts cultured in vitro display increased expression of intercellular adhesion molecule-1 and produce IL-6 plus IL-8 [27]. As indicated in murine fibroblast cells in vitro, hIL-17 induces production of IL-6 through activation of the transcription protein nuclear factor- $\kappa \mathrm{B}(\mathrm{NF}-\kappa \mathrm{B})$ [31]. Interestingly, hIL-17 also stimulates a human lung fibroblast lung cell line to produce IL-6 [33] and IL-6 release in human bronchial epithelial cells [33]. In addition, IL-17 also promotes granulopoiesis in mice [34]. For these reasons, IL-17 should have the capacity to mobilize airway neutrophils in vivo.

\section{Interleukin-17 induces the release of a neutrophil chemoattractant}

The neutrophil-recruiting capacity of IL-17 has recently been evaluated in vitro as well as in vivo [35]. It is now clear that hIL-17 increases the release of the neutrophil chemoattractant, IL-8 in a concentration-dependent fashion in a highly differentiated human airway epithelial cell line (16HBE) in vitro and that coincubation with a neutralizing anti-hIL-17 antibody blocks this IL-8 release [35]. IL-17 induces IL-8 release that is time-dependent in another human airway epithelial cell line (Calu-3) and in human umbilical vein endothelial cells (HUVEC's) [35]. This release of IL- 8 is probably due to de novo synthesis because hIL-17 increases the hIL- $8 / \beta$-actin mRNA ratios in $16 \mathrm{HBE}$ cells in vitro, as determined by reverse transcription polymerase chain reaction (fig. 1) [35]. Interestingly, hydrocortisone reduces the hIL-17-induced increase in IL-8, in human airway epithelial (16HBE plus Calu-3) cells as well as in HUVEC's. Cotreatment with a submaximally effective concentration of hIL-17 and hTNF- $\alpha$ results in a substantial potentiating effect on IL- 8 release from $16 \mathrm{HBE}$ cells [35]. The increase in IL-8 release caused by cotreatment with hIL-17 plus hTNF- $\alpha$ is even greater than that after treatment with a maximally effective concentration of hTNF- $\alpha$ alone, suggesting a synergistic stimulatory action of these two cytokines on the release of this potent neutrophil chemoattractant.

By taking conditioned medium from $16 \mathrm{HBE}$ cells cultured in vitro, and using this cell medium as a stimulus for isolated human neutrophils in a migration chamber in vitro, it has been shown that hIL-17 releases a chemotactic factor from these bronchial cells (fig. 2) [35]. Also, a neutralizing anti-hIL-8 antibody blocks this indirect effect of hIL-17, showing that IL-8 is the chemotactic factor involved. In contrast to conditioned medium from $16 \mathrm{HBE}$ cells stimulated with hIL-17, hIL-17 per se has no direct chemotactic effect on the migration of human neutrophils in vitro [35].

In a rat in vivo model, intratracheal instillation of hIL-17 significantly increases the absolute number of neutrophils in BAL fluid in a dose-dependent fashion (fig. 3) [36]. This increase in BAL neutrophil number is detected 4, 6 and $8 \mathrm{~h}$ after instillation of hIL-17, indicating a sustained effect. When rats are pretreated with a neutralizing antibody directed against hIL-17, a significant reduction in the IL-17-induced increase in BAL neutrophils is observed, underlining the specificity of the effect of IL-17 [35].

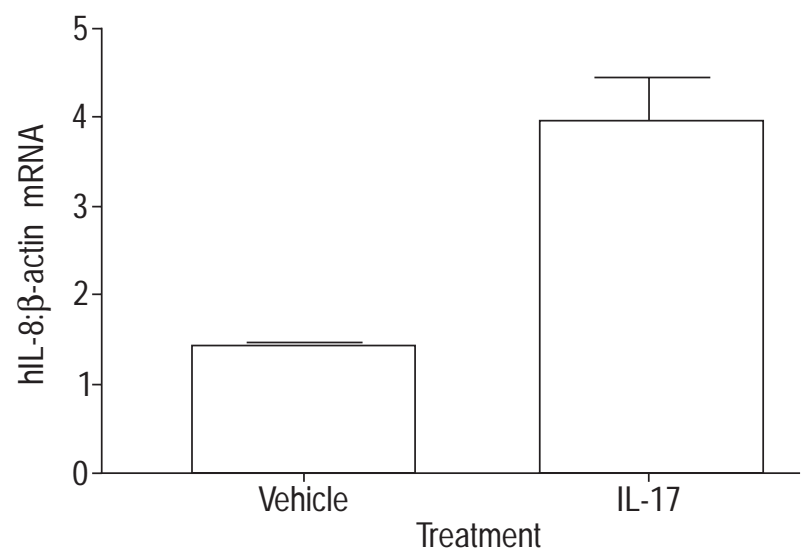

Fig. 1. - Expression of human interleukin (hIL)-8 messenger ribonucleic acid (mRNA) caused by hIL-17 $\left(1 \mu \mathrm{g} \cdot \mathrm{mL}^{-1}\right)$ in $16 \mathrm{HBE}$ cells after a 2-h stimulation. To standardize for variation in total ribonucleic acid levels, hIL- 8 mRNA expression was related to that of $\beta$-actin mRNA in electrophoresis gel using samples from duplicate experiments repeated 3 times. Values shown are mean \pm SEM (From [35].) 


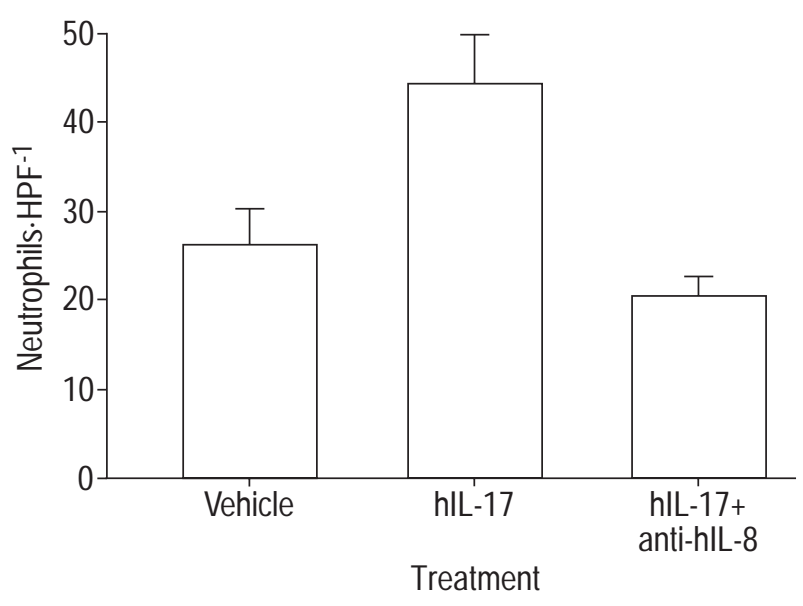

Fig. 2. - Human neutrophil migration caused by conditioned medium from 16HBE cells, treated with human interleukin (hIL)-17, in the chemotaxis multiwell chamber $\left(20-\mathrm{min}\right.$ incubation at $\left.37^{\circ} \mathrm{C}\right)$. Conditioned medium was obtained from $16 \mathrm{HBE}$ cells treated for $18 \mathrm{~h}$ with hIL-17 (1 $\mu \mathrm{g} \cdot \mathrm{mL}^{-1}$ ) or vehicle. Media from hIL-17-treated cells caused an increase in neutrophil migration versus vehicle. Coincubation with a monoclonal neutralizing anti-hIL-8 antibody $\left(15 \mathrm{~min}\right.$ at $\left.37^{\circ} \mathrm{C}\right)$ completely abolished this increase in neutrophil migration $(n=6)$. Values shown are mean $\pm \operatorname{SEM}$ and refer to the number of neutrophils per light microscope high-power field (HPF). (From [35].)

When comparing the effect of hIL-17 to that of rIL-1 $\beta$, a similar selectivity for neutrophil recruitment is observed [36]. In addition to this, it has now also been shown that selective neutrophil recruitment can be achieved by inducing overexpression of the IL-17 gene in mice airways [37]. Pretreatment with dexamethasone attenuates the hIL-17induced increase in BAL neutrophils, thereby pointing out a steroid-sensitive mechanism of action in vivo as well as in vitro [35]. This provides additional evidence for the universal anti-inflammatory effect of glucocorticoids and supports the idea that glucocorticoids act, in part, via inhibition of cytokine production [38].

The involvement of macrophage inflammatory protein-2 (MIP-2), the rat correlate to IL-8, has also been evaluated in rat airways in vivo, and the results confirm that hIL-17 instilled intratracheally increases the level of this CXC chemokine in BAL fluid [35]. The finding that pretreat-

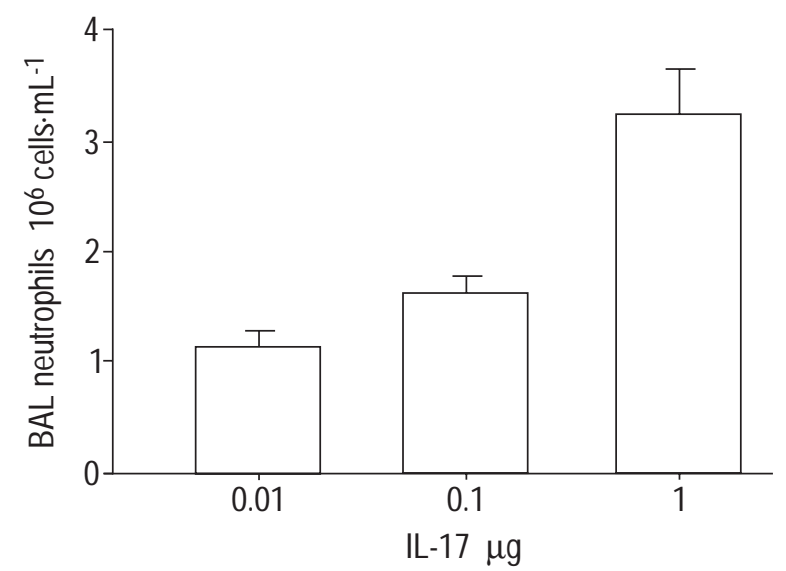

Fig. 3. - Dose-dependent effect of human interleukin (hIL)-17 (in 50 $\mu \mathrm{L}$ vehicle, 6-h incubation) given intratracheally on neutrophil number in bronchoalveolar lavage (BAL) fluid from Sprague-Dawley rats in vivo. Values shown are mean \pm SEM $(n=6)$. (From [36].) ment with a neutralizing antibody directed against mMIP2 significantly reduces the increase in BAL neutrophil number caused by hIL-17 provides additional evidence for the involvement of MIP-2 [35].

\section{Interleukin-17 acts, in part, via endogenous tachykinins}

The role of endogenous tachykinins in IL-17-induced airway neutrophilia has also been evaluated [36]. The results show that pretreatment with the peptidase inhibitors phosphoramidon plus captopril significantly enhances the number of neutrophils, but not that of other cell types, in BAL fluid from rats treated intratracheally with hIL-17 in vivo [36]. The relative magnitude of this effect is similar after corresponding treatment with rIL-1 $\beta$ [36]. In addition, pretreatment with phosphoramidon plus captopril does not significantly increase the neutrophil number in BAL fluid from rats treated with the vehicle of hIL-17 alone [36], thereby suggesting that IL-17 acts, in part, via release of endogenous tachykinins. Also, in rats given hIL-17 intratracheally, pretreatment with phosphoramidon enhances the neutrophil number in BAL fluid, whereas captopril does not [36]. Furthermore, pretreatment with the selective neurokinin (NK)-1 receptor antagonist SR 140333 significantly reduces the neutrophil number in BAL fluid after treatment with hIL-17, but this is not the case after pretreatment with the selective NK-2 receptor antagonist SR 48968 [36]. Taken together, these observations point out the NK-1 receptor as an important target for IL-17-induced release of endogenous tachykinins.

\section{Interleukin-17 activates neutrophils indirectly}

The capacity of IL-17 to activate airway neutrophils has been evaluated by analysing the activity of two neutrophilrelated enzymes in the cell-free component of BAL fluid supernatant from rat airways in vivo [39]. A moderate increase in elastase activity is present after intratracheal treatment with hIL-17 [36], and, in contrast, intratracheal instillation of rIL-1 $\beta$ alone does not cause a corresponding increase at an equally effective dose in terms of neutrophil recruitment. Rats pretreated with a threshold dose of rIL-1 $\beta$ display a significant enhancement of the elastase activity induced by hIL-17, whereas the BAL neutrophil count remains unaltered.

Quite similar to its effect on elastase activity, hIL-17 increases myeloperoxidase activity in cell-free BAL fluid supernatant, whereas IL-1 $\beta$ does not [39]. Pretreatment with a threshold dose of rIL-1 $\beta$ significantly enhances the myeloperoxidase activity induced by hIL-17, in analogy with its potentiating effect on hIL-17-induced elastase activity. Interestingly, in hIL-17-treated airways, the elastase activity correlates strongly with the myeloperoxidase activity, making a mechanistic association of elastase and myeloperoxidase release feasible.

By using a specific inhibitor of macrophage elastase (ethylene diamine tetra acetic acid) and neutrophil elastase 4-(z-Aminoethyl)-benzensulforylfluoride, respectively, a neutrophilic origin of the hIL-17-induced elastase activity has been ascertained [39]. In vehicle-treated rats, the inhibitor of macrophage elastase attenuates elastase activity in BAL fluid but this is not the case for the inhibitor of neutrophil elastase. In contrast, in hIL-17-treated rats, both the macrophage and the neutrophil elastase inhibitor 
attenuate elastase activity. Additional evidence in favour of hIL-17-induced neutrophil activation is provided by the strong correlation between the elastase activity sensitive to the neutrophil elastase inhibitor and myeloperoxidase activity [39]. There is no corresponding correlation between the elastase activity sensitive to the macrophage elastase inhibitor and myeloperoxidase activity.

In order to evaluate a hypothetical direct effect of IL-17, isolated rat neutrophils have been stimulated with hIL-17 in vitro [39]. However, the results show that hIL-17 causes no significant increase in myeloperoxidase activity in the conditioned cell medium of these neutrophils. The activation of airway neutrophils induced by hIL-17 is therefore likely to be an indirect effect.

\section{Conclusions}

Based upon the reviewed findings, it is concluded that IL-17, a cytokine released from certain activated T-lymphocytes, can play a pro-inflammatory role in the airways by recruiting and activating neutrophils (fig. 4). The effect of IL-17 on neutrophil recruitment is, at least in part, mediated via induced CXC chemokine release, probably achieved through de novo synthesis. The induced release of CXC chemokine can be potentiated by TNF- $\alpha$, a proinflammatory cytokine that is increased in obstructive airways disease [15], and, interestingly, is released by macrophages stimulated with IL-17 [40]. IL-17 can also exert its action via induced release of endogenous tachykinins, which contribute to neutrophil recruitment by acting on NK-1 receptors. Just like the effect on neutrophil recruitment, IL-17 can exert an indirect stimulatory effect on neutrophil activity in the airways. It is likely that IL-17 activates airway neutrophils through the induced release of neutrophil-activating cytokines, such as IL-6 and IL-8, which are known to be released from the bronchial epithelium and fibroblasts by IL-17 [33]. Interestingly, IL-1 $\beta$, another cytokine that is increased in obstructive airways disease [40], does potentiate the stimulatory effect of IL-17 on neutrophil activation. It can be speculated that IL-17 stimulates the release of IL-1 $\beta$ from airway macrophages and that this IL- $1 \beta$ potentiates the IL-17-induced release of IL- 6 and IL 8 in bronchial epithelial cells $[41,42]$.

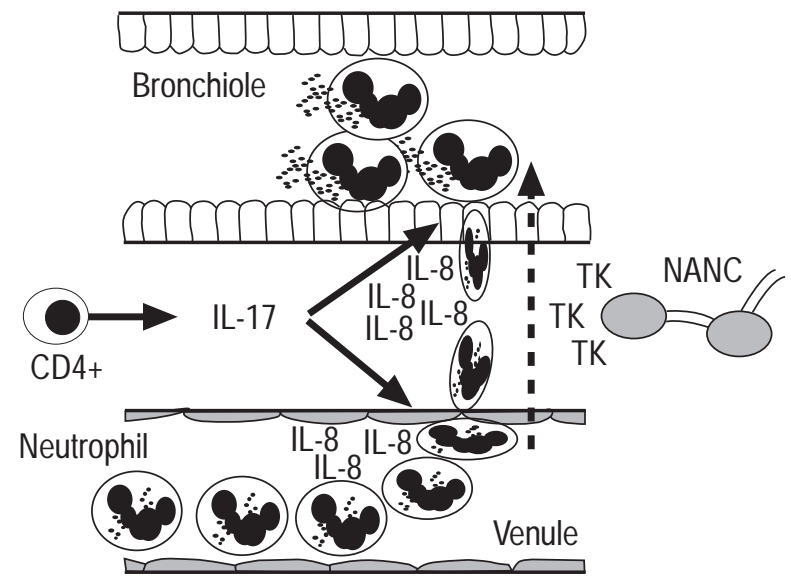

Fig. 4. - Schematic overview of the role of interleukin (IL)-17 in airway neutrophilia. TK: tachykinins; NANC: nonadrenergic noncholinergic nerves; CD4+: T-helper lymphocytes.
Even though it has now been shown that there is increased immunoreactivity for IL-17 in asthmatic airways [43], it is not known whether this increased immunoreactivity reflects the release of soluble biologically active IL-17 from CD4+ cells in the airway wall and/or lumen. Neither is it known whether the association of immunoreactivity for IL-17 and fibrotic areas in human bronchial tissue reflects a role of IL-17 in airway remodelling [43]. Yet another interesting issue is whether neutrophil activation and recruitment can account for the T-lymphocytedependent determination of airway responsiveness in vivo [44], and whether IL-17 is involved in this context.

The hypothesis that interleukin-17 serves as a mediator linking the activation of certain CD4+ cells to the recruitment and activation of airway neutrophils clearly needs further evaluation. Hopefully, an increased understanding of these mechanisms will reveal novel targets for pharmacotherapeutical intervention against exaggerated recruitment and activation of neutrophils in obstructive airways disease.

\section{References}

1. Fahy JV, Kim KW, Liu J, Boushey HA. Prominent neutrophilic inflammation in sputum from subjects with asthma excacerbation. J Allergy Clin Immunol 1995; 95: 843-852.

2. Laitinen LA, Laitinen A, Haahtela T. Airway mucosal inflammation even in patients with newly diagnosed asthma. Am Rev Respir Dis 1993; 147: 697-704.

3. Nocker RET, Schoonbrood DFM, van de Graaf EA, et al. Interleukin- 8 in airway inflammation in patients with asthma and chronic obstructive pulmonary disease. Int Arch Allergy Immunol 1996; 109: 183-191.

4. Saetta M, Turato G, Facchini FM, et al. Inflammatory cells in bronchial glands of smokers with chronic bronchitis. Am J Respir Crit Care Med 1997; 156: 1633-1639.

5. Hamid Q, Song Y, Kotsimbos TC, et al. Inflammation of small airways in asthma. J Allergy Clin Immunol 1997; 100: 44-51.

6. Lamblin C, Gosset P, Tillie-Leblond I, et al. Bronchial neutrophilia in patients with noninfectious status asthmaticus. Am J Respir Crit Care Med 1998; 157: 394-402.

7. Cortijo J, Villagrasa V, Pons R, et al. Bronchodilator and anti-inflammatory activities of glaucine: in vitro studies on human airway smooth muscle and polymorphonuclear leukocytes. Br J Pharmacol 1999; 127: 1641-1651.

8. Nadel JA, Stockley RA. Proteolytic enzymes and airway diseases. Eur Respir J 1998; 12: 1250-1251.

9. Schuster A, Ueki I, Nadel JA. Neutrophil elastase stimulates tracheal submucosal gland secretion that is inhibited by ICI 200,355. Am J Physiol 1992; 262: L86-L91.

10. Vignola AM, Bonanno A, Mirabella A, et al. Increased level of elastase and $\alpha_{1}$-antitrypsin in sputum of asthmatic patients. Am J Respir Crit Care Med 1998; 157: 505-511.

11. Watanabe M, Kaihatsu T, Miwa M, Maeda T. $\mathrm{Ca}^{2+}$ / calmodulin-dependent protein kinase II inhibitors potentiate superoxide production in polymorphonuclear leukocytes. J Pharm Pharmacol 1999; 51: 295-300.

12. Massion P, Lindén A, Inoue H, Mathy M, Grattan KM, Nadel JA. Dimethyl sulfoxide decreases interleukin-8mediated neutrophil recruitment in the airways. $A m J$ Physiol 1996; 271: L838-L843.

13. Jorens PG, Richman-Eisenstadt JBY, Housset BP, et al. Interleukin-8 induces neutrophil accumulation but not 
protease secretion in the canine trachea. Am $J$ Physiol 1992; 263: L708-L713.

14. Page SM, Gleich GJ, Roebuck KA, Thomas LL. Stimulation of neutrophil interleukin-8 production by eosinophil granule major basic protein. Am J Respir Cell Mol Biol 1999; 21: 230-237.

15. Keatings VM, Collins PD, Scott DM, Barnes PJ. Differences in interleukin- 8 and tumor necrosis factor- $\alpha$ in induced sputum from patients with chronic obstructive pulmonary disease or asthma. Am J Respir Crit Care Med 1996; 153: 530-534.

16. Volcano M, Alves Rosa MF, Minnucci FS, Chernavsky AC, Isturiz MA. $N$-formyl-methionyl-leucyl-phenylalanine ( $f \mathrm{MLP})$ inhibits tumor necrosis factor- $\alpha$ (TNF- $\alpha$ ) production in lipopolysaccharide (LPS)-stimulated human neutrophils. Clin Exp Immunol 1998; 113: 39-47.

17. Hessel EM, Van Oosterhout AJ, Van Ark I, et al. Development of airway hyperresponsiveness is dependent on interferon-gamma and independent of eosinophil infiltration. Am J Respir Crit Care Med 1997; 16: 325 334.

18. Lindén A. Increased interleukin- 8 released by $\beta$-adrenoceptor activation in human transformed bronchial epithelial cells. Br J Pharmacol 1996; 119: 402-406.

19. Xiu Q, Fujimura M, Nomura M, et al. Bronchial hyperreasponsiveness and airway neutrophil accumulation induced by interleukin- 8 and the effect of the thromboxane $\mathrm{A}_{2}$ antagonist S-1452 in guinea-pigs. Clin Exp Allergy 1995; 25: 51-59.

20. Perretti M, Ahluwalia A, Flower RJ, Manzini S. Endogenous tachykinins play a role in IL- $1 \beta$-induced neutrophil accumulation: involvement of NK1-receptors. Immunology 1993; 80: 73-77.

21. Walker C, Kaegi MK, Braun P, Blaser K. Activated T cells and eosinophilia in bronchoalveolar lavages from subjects with astma correlated with disease severity. $J$ Allergy Clin Immunol 1991; 88: 935-942.

22. Saetta M, Di Stefano A, Maestrelli P, et al. Activated Tlymphocytes and macrophages in bronchial mucosa of subjects with chronic bronchitis. Am Rev Respir Dis 1993; 147: 301-306.

23. Gavett SH, Chen X, Finkelman F, Wills-Karp M. Depletion of murine CD4+ T lymphocytes prevents antigen-induced airway hyperreactivity and pulmonary eosinophilia. Am J Respir Cell Mol Biol 1994; 10: 587-593.

24. Renzi PM, Yang JP, Diamantstein T, Martin JG. Effects of depletion of cells bearing the interleukin-2 receptor on immunoglobulin production and allergic airway responses in the rat. Am J Respir Crit Care Med 1996; 153: $1214-1221$.

25. Nakajima H, Iwamoto I, Tomoe S, et al. CD4+ T-lymphocytes and interleukin-5 mediate antigen-induced eosinophil infiltration into the mouse trachea. Am Rev Respir Dis 1992; 146: 374-377.

26. Fossiez F, Banchereau J, Murray R, Van Kooten C, Garrone P, Lebecque S. Interleukin-17. Intern Rev Immunol 1998; 16: 541-551.

27. Yao Z, Painter SL, Fanslow WC, et al. Human IL-17: a novel cytokine derived from $\mathrm{T}$ cells. J Immunol 1995; 155: 5483-5486.

28. Spriggs MK. Interleukin-17 and its receptor. $J$ Clin Immunol 1997; 17: 366-369.
29. Aarvak T, Chabaud M, Miossec P, Natvig JB. IL-17 is produced by some proinflammatory Th1/Th0 cells but not Th2 cells. J Immunol 1999; 162: 1246-1251.

30. Shin H, Benbernou N, Esnault S, Guenounou M. Expression of IL-17 in human memory CD45RO+ T lymphocytes and its regulation by protein kinase A pathway. Cytokine 1999; 11: 257-266.

31. Yao Z, Spriggs M, Derry J, et al. Molecular characterisation of the human interleukin (IL)-17 receptor. Cytokine 1997; 9: 794-800.

32. Yao Z, Fanslow FC, Seldin MF, et al. Herpesvirus Saimiri encodes a new cytokine, IL-17, which binds to a novel cytokine receptor. Immunity 1995; 3: 811-821.

33. Fossiez F, Djossou O, Chomarat $\mathrm{P}$, et al. T cell interleukin-17 induces stromal cells to produce proinflammatory and hematopoietic cytokines. J Exp Med 1996; 183: 2593-2603.

34. Schwarzenberger P, La Russa V, Miller A, et al. IL-17 stimulates granulopoesis in mice: use of an alternate genetherapy derived method for in vivo evaluation of cytokines. J Immunol 1998; 161: 6383-6389.

35. Laan M, Cui ZH, Hoshino $\mathrm{H}$, et al. Neutrophil recruitment by human IL-17 via $\mathrm{C}-\mathrm{X}-\mathrm{C}$ chemokine release in the airways. J Immunol 1999; 162: 2347-2352.

36. Hoshino H, Lötvall J, Skoogh BE, Lindén A. Neutrophil recruitment by IL-17 into rat airways in vivo: role of tachykinins. Am J Respir Crit Care Med 1999; 159: 14231428.

37. Ye P, Garvey P, Huang D, et al. IL-17 increases lung immunity and improves lung host defense against $K$. Pneumonie infection. Am J Respir Crit Care Med 1999; 159: A92.

38. Barnes PJ, Pedersen S. Efficacy and safety of inhaled steroids in asthma. Am Rev Respir Dis 1993; 148: S126.

39. Hoshino H, Laan M, Sjöstrand M, Lötvall J, Skoogh BE, Lindén A. Increased elastase and myeloperoxidase activity associated with neutrophil recruitment by IL-17 in airways in vivo. J Allergy Clin Immunol 2000; 105: 143149.

40. Tillie-Leblond I, Pugin J, Marquette $\mathrm{CH}$, et al. Balance between proinflammatory cytokines and their inhibitors in bronchial lavage from patients with status asthmaticus. Am J Respir Crit Care Med 1999; 159: 487-494.

41. Jovanovic DV, Di Battista JA, Martel-Pelletire J, et al. IL-17 stimulates the production and expression of proinflammatory cytokines IL1- $\beta$ and TNF- $\alpha$ by human macrophages. J Immunol 1998; 160: 3513-3521.

42. Bedard M, McLure CD, Schiller NL, Francoer C, Cantin A, Denis M. Release of interleukin-8, interleukin-6, and colony stimulating factor by upper airway epithelial cells: implications for cystic fibrosis. Am J Cell Mol Biol 1993; 9: 455-462.

43. Molet S, Chakir J, Lavigne F, Olivenstein R, Taha R, Hamid QA. Increased expression of IL-17 in asthma and chronic sinusitis: possible association with tissue remodelling. J Allergy Clin Immunol 1999; 103: Suppl. 107, A407.

44. De Sanctis GT, Itoh A, Green FHY, et al. T-lymphocytes regulate genetically determined airway hyperresponsiveness in mice. Nature Med 1997; 4: 460-462. 